\section{Ultrasonographic findings of Kikuchi cervical lymphadenopathy in children}

\author{
Ji Young Kim", Hyunju Lee², Bo La Yun ${ }^{1}$ \\ Departments of ${ }^{1}$ Radiology and ${ }^{2}$ Pediatrics, Seoul National University Bundang Hospital, \\ Seongnam, Korea
}

Purpose: The purpose of this study was to analyze the ultrasonographic (USG) findings of Kikuchi cervical lymphadenopathy in pediatric patients.

Methods: Between April 2007 and September 2016, 84 children (42 male and 42 female; mean \pm standard deviation age, $12.9 \pm 3.2$ years; range, 5 to 18 years) confirmed with Kikuchi disease were enrolled. Clinical findings and USG findings of Kikuchi cervical lymphadenopathy were retrospectively reviewed. Localized symptoms, systemic symptoms, and laboratory findings including the white blood cell count, erythrocyte sedimentation rate (ESR), and C-reactive protein (CRP) were analyzed. An analysis of the USG findings included evaluation of the location, size, and presence of intranodal abscess; intranodal calcification; perinodal fat swelling; localized fluid collection; and loss of nodal echogenic hilum.

Results: Among the patients, 49 (58\%) showed localized tenderness at the cervical lymphadenopathy. Fever was present in 55 (66\%), while 27 (32\%) had prolonged fever. of 74 with lab results, $54(73 \%)$ had leukopenia but none had leukocytosis. Among the same 74 , there was a high ESR (>50 mm/hr) in $10(14 \%)$ and a high CRP level (>5 mg/dL) in seven ( $9 \%)$. The USG findings of most of the patients $(n=72,86 \%)$ showed unilateral neck involvement, especially in the left side neck (45 of $72,63 \%$ ). The most common site of Kikuchi lymphadenopathy involvement was the area at cervical lymph node level $V$, at the posterior triangle $(n=77,92 \%)$. Conglomerated nodal distribution ( $n=57,68 \%)$, preserved central nodal echogenic hilum $(n=84$, $98 \%$ ), and perinodal fat swelling ( $n=55,65 \%$ ) were common USG findings in the children with Kikuchi. In addition, multiple cervical lymph nodes showed a relatively even size distribution $(n=73,87 \%)$.

Conclusion: The common USG findings of Kikuchi disease in the pediatric population of our study were multiple conglomerated unilateral cervical lymphadenopathy showing perinodal fat swelling and even size distribution.

Keywords: Histiocytic necrotizing lymphadenitis; Ultrasonography; Pediatrics

\section{Introduction}

Kikuchi disease, also known as Kikuchi-Fujimoto disease or histiocytic necrotizing lymphadenitis is a rare, benign, self-limiting cause of cervical lymphadenopathy $[1,2]$. Kikuchi disease has various clinical features with a wide spectrum from absence of systemic symptoms to severe systemic

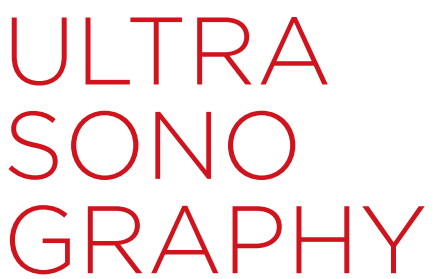

ORIGINAL ARTICLE

https://doi.org/10.14366/usg. 16047 pISSN: 2288-5919 • elSSN: 2288-5943 Ultrasonography 2017;36:66-70

Received: September 29, 2016

Revised: December 1, 2016

Accepted: December 2, 2016

Correspondence to:

Ji Young Kim, MD, Department of Radiology, Seoul National University Bundang Hospital, 82 Gumi-ro

173beon-gil, Bundang-gu, Seongnam 13620, Korea

Tel. +82-31-787-7609

Fax. +82-31-787-4011

E-mail: poohahe@gmail.com

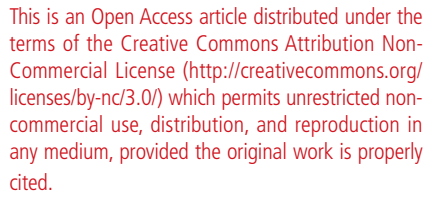

Copyright @ 2017 Korean Society of Ultrasound in Medicine (KSUM)

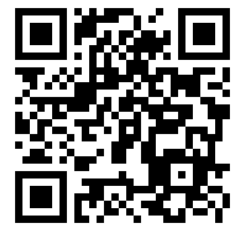

How to cite this article:

Kim JY, Lee H, Yun BL. Ultrasonographic findings of Kikuchi cervical lymphadenopathy in children. Ultrasonography. 2017 Jan;36(1): 66-70. 
symptoms such as prolonged fever, arthralgia, and hemophagocytic lymphohistiocytosis [2-6]. Among these various clinical features of Kikuchi disease, cervical lymphadenopathy is the one of the most common clinical findings [7].

No specific laboratory tests are available for the diagnosis of Kikuchi disease. Because Kikuchi disease is a benign and selflimiting disease, early proper diagnosis is important in order to avoid unnecessary evaluations for various other causes of cervical lymphadenopathy and unnecessary treatment such as antibiotics. Kikuchi disease could be confirmed by histopathological findings of lymph node (LN) biopsy under ultrasonographic (USG) guidance [8]. However, biopsy is an invasive procedure with several potential complications and is difficult to conduct in children due to the need for admission or sedation. The delay in diagnosis could lead to frequent hospital visits, additional costs for evaluation, and school absenteeism.

Although several studies have reported the computed tomography (CT) or magnetic resonance imaging (MRI) findings of Kikuchi cervical lymphadenopathy $[7,9,10], C T$ and MRI are not the usual imaging modalities used in children due to the radiation hazard and the need for sedation. Instead, USG imaging is the most commonly used modality for evaluating cervical lymphadenopathy in children. However, to our knowledge, no large-scale analysis of USG findings of Kikuchi cervical lymphadenopathy in children has been reported.

Therefore, the purpose of this study is to describe the USG findings of cervical lymphadenopathy due to Kikuchi disease in children.

\section{Materials and Methods}

\section{Patient Population}

This study was approved by our Institutional Review Board, and informed consent was waived due to the retrospective design. We reviewed the electronic medical records and histopathological records of patients at our institution between April 2007 and September 2016. A total of 530 children $\leq 18$ years old had undergone an ultrasonographically guided gun biopsy for cervical lymphadenopathy. Of them, 87 children were histopathologically confirmed with Kikuchi disease. Among them, three children who did not undergo a full USG examination for re-evaluating both cervical chains were excluded. Ultimately, 84 children histopathologically confirmed with Kikuchi disease were enrolled in this study.

\section{Analysis of Clinical Findings}

Clinical characteristics, associated symptoms, and laboratory findings were collected from the medical records. Age and sex; localized symptoms including $L N$ tenderness or erythema or a sensation of heat; systemic symptoms such as fever; and physical findings including hepatomegaly or splenomegaly were all analyzed. To evaluate the incidence of prolonged fever lasting more than 2 weeks, we also evaluated the duration of the fever. For the analysis of laboratory findings, we excluded 10 patients whose laboratory findings were unavailable. Therefore, the total number of patients included in the laboratory finding analysis was 74 . The laboratory findings for evaluating leukopenia (white blood cell count $<4,000 /$ $\mathrm{mm}^{3}$ ), leukocystosis (white blood cell count $>10,000 / \mathrm{mm}^{3}$ ), high erythrocyte sedimentation rate (ESR, $>50 \mathrm{~mm} / \mathrm{h})$, and high level of C-reactive protein (CRP, $>5 \mathrm{mg} / \mathrm{dL}$ ) were also analyzed.

\section{Ultrasonography and Ultrasonographically Guided Gun Biopsy}

Ultrasonography was performed using a single ultrasonography system, the iU22 (Philips Healthcare, Bothell, WA, USA) equipped with a linear high-frequency probe (5-14 MHz). Ultrasonographically guided gun biopsies were performed using a disposable, 18-gauge, double-action, spring-activated needle (1.1- or 1.6-cm excursion, TSK Ace-cut, Create Medic, Yokohama, Japan). After local anesthesia with $1 \%$ lidocaine, the end of the biopsy needle was advanced into the cortex of the LN using a free-hand technique, after which the stylet and cutting cannula of the needle were fired sequentially. The need for sedation was determined according to the patient's cooperation.

\section{Image Analysis}

Two radiologists (J.Y.K. and B.L.Y.) retrospectively reviewed the USG findings and drew conclusions by consensus. The following USG findings were analyzed: (1) location of the cervical lymphadenopathy (bilateral vs. unilateral and the level of cervical LNs); (2) distribution of the cervical lymphadenopathy (conglomerated vs. multiple separated); (3) the presence of intranodal gross abscess and calcification; (4) the loss of central echogenic nodal hilum; (5) the presence of perinodal fat swelling; (6) the presence of loculated fluid collection; and (7) size distribution of LNs. The author also measured the long diameter (LD) and short diameter (SD) of the largest cervical $L N$, and the ratios of $L D$ to SD of the lesions (LD/SD) were calculated. We defined an enlarged LN as an LN with an SD of more than $10 \mathrm{~mm}$.

\section{Results}

\section{Clinical Findings}

The patient's clinical findings are summarized in Table 1. The mean age of 84 children with Kikuchi disease was $12.9 \pm 3.2$ years (standard deviation), and the age range was $5-18$ years old. The 
results showed even sex distribution of Kikuchi disease, with a female to male ratio of $1: 1$. In the children aged 10 or younger $(n=19)$, the sex ratio was 13:6. Of all 84 patients, 49 patients (58\%) had localized tenderness at the cervical lymphadenopathy. However, only one (1\%) child showed localized erythema and 11 children $(13 \%)$ had a sensation of heat. Fever was present in $55(66 \%)$, and the median duration of fever was 9 days (interquartile range, 0.25 to 17.0). In addition, 27 children (32\%) showed prolonged fever (more than a 2-week duration). A few children presented with hepatomegaly $(n=2,2 \%)$ or splenomegaly $(n=4,5 \%)$.

In the 74 patients with laboratory findings, 54 (73\%) had leukopenia. The remaining 20 of 74 patients (27\%) had a white blood cell count in normal range. No one was found to have leukocytosis. A high ESR (>50 mm/hr) was found in 10 patients $(14 \%)$ and a high CRP level (>5 mg/dL) in seven ( $9 \%)$.

Table 1. Clinical and laboratory finding of Kikuchi disease in children

\begin{tabular}{|c|c|}
\hline Clinical and laboratory finding & No. of patients $(\%)(n=84)$ \\
\hline Age (yr) & $12.9 \pm 3.2(5-18)$ \\
\hline \multicolumn{2}{|l|}{ Sex } \\
\hline Female & $42(50)$ \\
\hline Male & $42(50)$ \\
\hline \multicolumn{2}{|l|}{ Localized symptoms } \\
\hline LN tenderness & $49(58)$ \\
\hline LN erythema & $1(1)$ \\
\hline LN sense of heat & $11(13)$ \\
\hline \multicolumn{2}{|l|}{ Systemic symptoms } \\
\hline Fever & $55(66)$ \\
\hline Prolonged fever ( $\geq 2$ wk) & $27(32)$ \\
\hline Hepatomegaly & $2(2)$ \\
\hline Splenomegaly & $4(5)$ \\
\hline \multicolumn{2}{|l|}{ Laboratory findings $s^{\text {a) }}$} \\
\hline $\mathrm{WBC}\left(4 \times 10^{3} / \mathrm{mm}^{3}\right)$ & $3.3 \pm 1.5(1.0-6.9)$ \\
\hline Leukopenia (WBC <4,000/mm³) & $54(73)$ \\
\hline $\mathrm{ESR}(\mathrm{mm} / \mathrm{hr})$ & $32 \pm 21(3-116)$ \\
\hline High ESR (>50 mm/hr) & $10(14)$ \\
\hline $\operatorname{CRP}(\mathrm{mg} / \mathrm{dL})$ & $2.1 \pm 3.1(0.01-17)$ \\
\hline High CRP ( $>5 \mathrm{mg} / \mathrm{dL})$ & $7(9)$ \\
\hline
\end{tabular}

Values are presented as numbers of patients (\%) or mean \pm standard deviation (range).

LN, lymph node; WBC, white blood cell count; ESR, erythrocyte sedimentation rate; CRP, C-reactive protein.

a)Patients whose laboratory findings were unavailable for analysis were excluded: total number for laboratory findings $=74$.

\section{USG Findings}

The USG findings of Kikuchi disease in children are summarized in Table 2. All 84 children had multiple LNs. Most of the patients ( $n=72$, $86 \%$ ) showed unilateral neck involvement. Among them, left side neck involvement (45 of $72,63 \%$ ) was more common than right side. Bilateral neck involvement was found in $12(14 \%)$. The most common site of Kikuchi lymphadenopathy involvement was the area at the cervical LN level $\mathrm{V}$, posterior triangle $(n=77,92 \%)$. Other frequently affected node levels were level IV ( $n=63,75 \%)$, III $(n=63$, $75 \%)$, and II $(n=60,71 \%)$. Conglomerated nodal distribution $(n=57$, $68 \%$ ) was more common than separate LN (Fig. 1). According to our results, most cervical LN of Kikuchi disease preserved their central echogenic hilum on ultrasonography $(n=82,98 \%)$. Perinodal fat swelling $(n=55,65 \%)$ was a relatively common USG finding in Kikuchi children (Fig. 2). Because perinodal fat swelling was caused by perinodal edema, the echogenicity of the swollen perinodal fat was increased on USG imaging. No cases showed intranodal abscess, intranodal calcification, or perinodal fluid collection on ultrasonography. Multiple cervical LN showed a relatively even size distribution $(n=73,87 \%)$. The mean SD and LD of the largest cervical LN were $8.5 \mathrm{~mm}$ (range, 4 to $21 \mathrm{~mm}$ ) and $18.7 \mathrm{~mm}$ (range,

Table 2. USG findings of Kikuchi disease in children

\begin{tabular}{lc}
\hline \multicolumn{1}{c}{ USG finding } & No. of patients (\%) $(\mathrm{n}=84)$ \\
\hline Location & $72(86)$ \\
Unilateral & 27 \\
Right & 45 \\
Left & $12(14)$ \\
Bilateral & \\
Distribution & $57(68)$ \\
Conglomerated & $27(32)$ \\
Separated & 0 \\
Intranodal abscess & 0 \\
Intranodal calcification & $2(2)$ \\
Loss of central echogenic hilum & $55(65)$ \\
Perinodal fat swelling & 0 \\
Perinodal fluid collection & \\
Size distribution of cervical LN & $73(87)$ \\
Even & $11(13)$ \\
Uneven & \\
Size of largest cervical LN & $8.5 \pm 3.2(4-21)$ \\
SD (mm) & $18.7 \pm 6.4(7-40)$ \\
LD (mm) & $0.5 \pm 0.1(0.2-0.7)$ \\
SD/LD ratio & \\
\hline
\end{tabular}

Values are presented as numbers of patients (\%) or mean \pm standard deviation (range).

USG, ultrasonographic; LN, lymph node; SD, short diameter; LD, long diameter. 


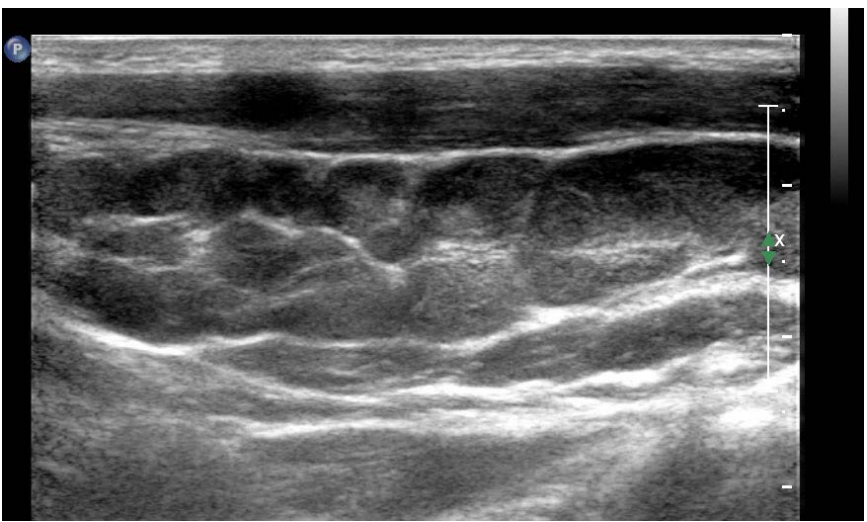

Fig. 1. Kikuchi cervical lymphadenopathy in a 10-year-old male. Longitudinal sonogram shows multiple well-defined, conglomerated lymph nodes in right cervical lymph node levels II, III, IV, and V. The size of the cervical lymph nodes shows even distribution. It should be noted that the central echogenic hilum of the cervical lymph nodes is preserved.

7 to $40 \mathrm{~mm}$ ), respectively. Among the patients, 22 had multiple enlarged LNs. A large number of patients (62 of $84,74 \%$ ) did not have any LNs enlarged to a diameter of more than $10 \mathrm{~mm}$. The mean SD to $L D$ ratio was $0.5 \pm 0.1$ (standard deviation).

\section{Discussion}

Kikuchi disease is an idiopathic disease usually characterized by self-limiting painful cervical lymphadenopathy. Cervical lymphadenopathy of Kikuchi disease has a tendency to be located in the posterior cervical region $[4,11]$. This study also found that the posterior triangle $(n=77,92 \%)$ was the most common site of involvement. Therefore, when posterior cervical lymphadenopathy occurs in children, Kikuchi disease should be included in the differential diagnosis. Regarding age, we found that Kikuchi disease in children commonly occurred in early adolescence. In our study, the sex distribution of Kikuchi disease was even, with a male to female ratio of 1:1. However, when the age was limited to 10 years or less, the ratio of boys to girls was 13:6, showing a male predominance. This result is consistent with previous reports on a pediatric population [1]. On the other hand, in the adult population, female predominance is a characteristic finding of Kikuchi disease [12].

Fever is one of the common systemic symptoms of Kikuchi disease, but its severity varies [1]. According to our results, persistent prolonged fever was observed in $32 \%$ up to 2 weeks, but fever was not observed in $34 \%$. The systemic symptoms of Kikuchi disease include not only fever; in fact, the range of presentation and severity is broad. It is often difficult to diagnose Kikuchi disease with certain clinical findings. It can be difficult even with laboratory findings.

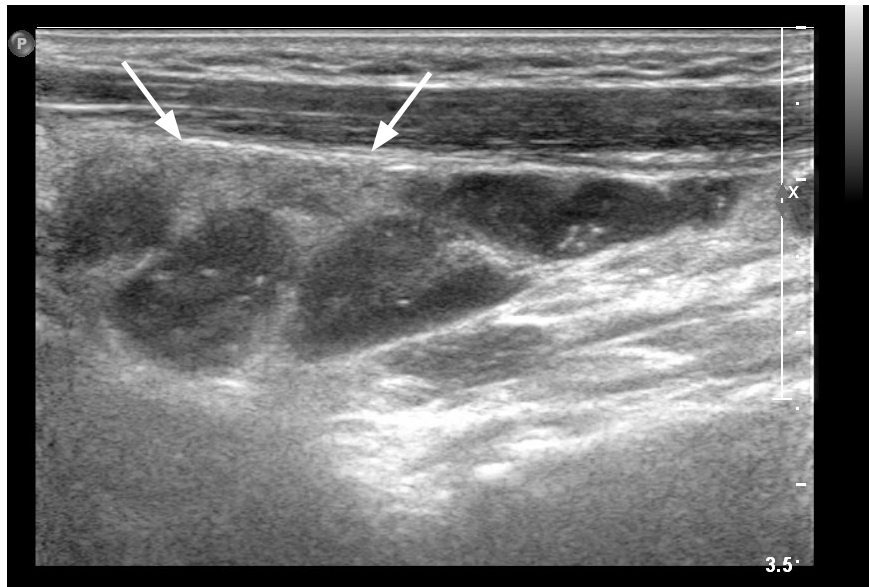

Fig. 2. Kikuchi cervical lymphadenopathy in a 5-year-old male. A longitudinal sonogram shows multiple conglomerated lymph nodes in the left cervical jugular chain. The perinodal fat swelling with increased echogenicity (arrows) should be noted.

Although leukopenia is helpful in the diagnosis of Kikuchi disease because of its distinctive laboratory findings, findings of the ESR or CRP may not contribute much to the differential diagnosis because they can be associated with other inflammatory diseases.

Radiological findings can be helpful in diagnosing Kikuchi disease when the diagnosis is unclear based on clinical findings and laboratory findings. Ultrasonography, in particular, is a good option for diagnostic radiologic examination of cervical lymphadenopathy in pediatric patients. It presents no radiation hazard and no need for additional sedation because of the short scan time. In a previous study of 175 adults with Kikuchi disease, perinodal echogenicity was reported as a characteristic finding of Kikuchi disease [13]. According to our results performed in children, perinodal fat swelling with increased fat echogenicity $(n=55,65 \%)$ was a common finding in Kikuchi disease, and this was consistent with previous studies. This is a finding that can be seen in other benign cervical lymphadenopathies such as infectious lymphadenitis. However, considering the various USG findings (such as unilateral conglomerated distribution, even size distribution for cervical LN, no intranodal abscess, and no intranodal calcification) that are more common in Kikuchi disease cases in this study, ultrasonography should still be helpful for identifying Kikuchi disease.

The USG findings of Kikuchi disease revealed in this study will help to narrow the range of differential diagnosis by examination in pediatric cases presented with cervical lymphadenopathy with fever. Early diagnosis using USG findings can also reduce unnecessary diagnostic and treatment costs.

This study is a retrospective study in which clinical information was drawn only from medical records, so it was difficult to carry out 
research with only incomplete clinical information. The study was also limited by the fact that the color Doppler exam images could not be analyzed because few of the USG exams included color Doppler imaging.

In conclusion, the common USG findings of Kikuchi disease in our pediatric population study were multiple conglomerated unilateral cervical lymphadenopathy showing perinodal fat swelling and even size distribution.

ORCID: Ji Young Kim: http://orcid.org/0000-0003-1466-2112; Hyunju Lee: http://orcid.org/0000-0003-0107-0724; Bo La Yun: http://orcid.org/0000-00025457-7847

\section{Conflict of Interest}

No potential conflict of interest relevant to this article was reported.

\section{References}

1. Seo JH, Shim HS, Park JJ, Jeon SY, Kim JP, Ahn SK, et al. A clinical study of histiocytic necrotizing lymphadenitis (Kikuchi's disease) in children. Int J Pediatr Otorhinolaryngol 2008;72:1637-1642.

2. Chuang $\mathrm{CH}$, Yan DC, Chiu CH, Huang YC, Lin PY, Chen CJ, et al. Clinical and laboratory manifestations of Kikuchi's disease in children and differences between patients with and without prolonged fever. Pediatr Infect Dis J 2005;24:551-554.

3. Dorfman RF, Berry GJ. Kikuchi's histiocytic necrotizing lymphadenitis: an analysis of 108 cases with emphasis on differential diagnosis.
Semin Diagn Pathol 1988:5:329-345.

4. Lee $K Y$, Yeon $Y H$, Lee BC. Kikuchi-Fujimoto disease with prolonged fever in children. Pediatrics 2004;114:e752-e756.

5. Kim YM, Lee YJ, Nam SO, Park SE, Kim JY, Lee EY. Hemophagocytic syndrome associated with Kikuchi's disease. J Korean Med Sci 2003;18:592-594.

6. Chen JS, Chang KC, Cheng CN, Tsai WH, Su IJ. Childhood hemophagocytic syndrome associated with Kikuchi's disease. Haematologica 2000;85:998-1000.

7. Kwon SY, Kim TK, Kim YS, Lee KY, Lee NJ, Seol HY. CT findings in Kikuchi disease: analysis of 96 cases. AJNR Am J Neuroradiol 2004;25:1099-1102.

8. Kang HM, Kim JY, Choi EH, Lee HJ, Yun KW, Lee H. Clinical characteristics of severe histiocytic necrotizing lymphadenitis (Kikuchi-Fujimoto disease) in children. J Pediatr 2016;171:208-212.

9. Na DG, Chung TS, Byun HS, Kim HD, Ko YH, Yoon JH. Kikuchi disease: CT and MR findings. AJNR Am J Neuroradiol 1997;18:1729-1732.

10. Fulcher AS. Cervical lymphadenopathy due to Kikuchi disease: US and CT appearance. J Comput Assist Tomogr 1993;17:131-133.

11. Chen CK, Low Y, Akhilesh M, Jacobsen AS. Kikuchi disease in Asian children. J Paediatr Child Health 2006;42:104-107.

12. Kucukardali Y, Solmazgul E, Kunter E, Oncul O, Yildirim S, Kaplan M. Kikuchi-Fujimoto disease: analysis of 244 cases. Clin Rheumatol 2007;26:50-54.

13. Yoo JL, Suh SI, Lee YH, Seo HS, Kim KM, Shin BK, et al. Gray scale and power Doppler study of biopsy-proven Kikuchi disease. J Ultrasound Med 2011;30:957-963. 